\title{
3D Microstructure Characterization of a Silicon Based Anode Material on Different Length Scales suitable for Storage Applications
}

\author{
T. Vorauer ${ }^{1}$, P. Kumar ${ }^{2}$, F. F. Chamasemani ${ }^{1}$, J. Rosc ${ }^{1}$, B. Fuchsbichler ${ }^{3}$, S. Koller ${ }^{3}$, L. Helfen ${ }^{4,5}$, P.H. \\ Jouneau $^{2}$, S. Lyonnard ${ }^{2}$ and R. Brunner ${ }^{1}$ \\ 1. Materials Center Leoben, Forschung GmbH, Leoben, Austria. \\ 2. Univ. Grenoble Alpes, CEA, DRF/INAC/MEM, Grenoble, France. \\ 3. Varta Micro Innovation GmbH, Graz, Austria. \\ 4. Karlsruhe Institute of Technology, Karlsruhe, Germany. \\ 5. European Synchrotron Radiation Facility, Grenoble, France.
}

Lithium-based technology plays an important role in the context of energy storage systems [1]. However, there are still several shortcomings with respect to lithium (Li)-ion batteries. Mainly those challenges arise due to material deficiencies, which lead to a limited lifetime and the still high-energy costs per $\mathrm{kWh}$. In this context, new material concepts and processing steps need to be investigated. A highly promising approach in this respect displays the use of silicon based anode materials. Here, still several challenges from the scientific but also industrial application point of view arises. Major points in this context comprise the impact (1) of various loading cycles, with respect to degrading mechanism and reliability as well as (2) of the storage capacity.

Since the microstructure of the material relates to the mechanical, electrical and chemical properties of the material, it has a direct impact on the cell performance. Therefore, the 3D characterization of the microstructure itself and the study of its architecture is very important. Nevertheless, this is demanding due to the different length scales existing in the complex material mix ranging from $\mu \mathrm{m}$, down to $\mathrm{nm}$ on e.g. Si-particle-level. Therefore, a multi method approach with reliable and efficient image analysis algorithm is necessary to provide conclusive 3D information on the different length scales. This approach should provide improved design guidelines for the used materials as well as for the cells.

In this work [2], we discuss the use of a multi-scale approach suitable for the analysis of Si-based pristine and cycled anode material relevant for industrial applications. In order to meet the complexity of the material on different length scales we use (1) micro-x-ray computed tomography, (2) micro-synchrotron tomography, (3) nano-FIB-tomography as well as (4) STEM together with energy dispersive x-ray spectroscopy (EDS).

To investigate larger scales $(\geq 1 \mu \mathrm{m})$, we use x-ray computed tomography (XCT). Here, we perform measurements on anode samples with a size of about $0.5 \times 1 \mathrm{~mm}^{2}$. We used for the XCT measurements a voxel size of about $\mathrm{V}_{\mathrm{x}}=\mathrm{V}_{\mathrm{y}}=\mathrm{V}_{\mathrm{z}}=350 \mathrm{~nm}$. This provides the possibility to examine the overall distribution of the matrix material (graphite) as well as the active material (Si-particles). At this scale, we focused in particular on the analysis of the distribution of agglomerated Si-particles and the continuous Si phases. The analyzable volume of interest (VOI) is about $17 \times 655 \times 420 \mu^{3}$. In detail, we analyzed properties from the Si-particles like radius, surface area and next-neighbor distance in the 3D volume. To gain information about the Si-particle percentage and its impact on the microstructure we also investigated anode samples with different Si-weight percentages $(7 \%, 15 \%, 20 \%, 25 \%$ and $30 \%)$.

To complement the XCT results, we have made in addition synchrotron measurements, which were performed at the ID19 beamline of the ESRF. The resolution is about $650 \mathrm{~nm}$ pixel size. Here, we investigated the microstructure changes of pristine as well as of cycled samples with a higher resolution. The samples were cycled up to 300 cycles. The VOI is about $35 \times 500 \times 600 \mu \mathrm{m}^{3}$. 
Considering the complexity of the anode electrode that contains features ranging from tens of $\mu \mathrm{m}$ up to $\mathrm{nm}$ scale, a novel correlative approach has been applied by combining focused ion beam nano-tomography (FIBSEM) and scanning transmission electron microscopy (STEM) together with an energy dispersive x-ray spectroscopy (EDS). With this approach, we cover a large length scale ranging from several tens of $\mu \mathrm{m}$ to (sub) nm scale.

$3 \mathrm{D}$ reconstruction of the pristine and the cycled electrodes has been performed by acquiring $2 \mathrm{D}$ slices with a voxel size of $\mathrm{V}_{\mathrm{x}}=\mathrm{V}_{\mathrm{y}}=8 \mathrm{~nm}$ and $\mathrm{V}_{\mathrm{z}}=16 \mathrm{~nm}$. The final volume of the electrode was $\sim 40 \mathrm{x} 40 \times 15 \mu \mathrm{m}^{3}$ with a pixel size of $8 \mathrm{~nm}$ in the $\mathrm{x}$ and y plane while the slice thickness (z-depth spacing) was $16 \mathrm{~nm}$. The goal of the 3D FIB-SEM nano-tomography was to: (i) measure quantitatively the porosity within and between the graphite particles, (ii) to observe the evolution (morphology) of the anode electrode at large cycling (typically 300) at both stages i.e., lithiated/delithiated and, (iii) finally, to determine the chemical repartition of the various phases.

Furthermore, TEM-lamella of the anode samples have been prepared in cross-sections and characterized using a Cs-corrected STEM operated at $200 \mathrm{kV}$ and equipped with a windowless EDX detector. The chemical mapping of the various phases was obtained by generating EDS maps with a lateral resolution of $\sim 30 \mathrm{~nm}$. To better understand the aging mechanism, we investigated the structural (morphology and crystalline quality) and chemical evolution of the different phases such as Si particles, graphite and the formation of the Solid electrolyte interphase (SEI) layer during the charge/discharge process of the electrode.

\section{References:}

[1] European Energy Storage Technology Development Roadmap Towards 2030 EERA (2013). http://www.eera-set.eu/wp-content/uploads/148885-EASE-recommendations-Roadmap-04.pdf

[2] This work has received funding from the European Union (EU) under the Horizon 2020 research and innovation program (grant agreement no. 685716 "SINTBAT").

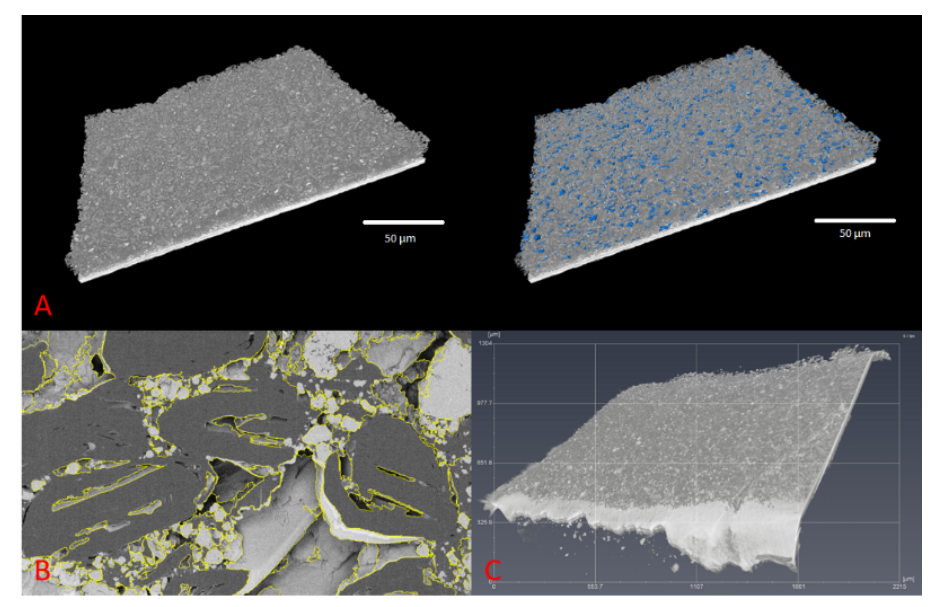

Figure 1. (A) Synchrotron images of the pristine anode material. The bright layer at the bottom is the copper substrate. Left image shows the selected ROI $\left(600 \times 500 \times 35 \mu \mathrm{m}^{3}\right)$ with a voxel size of $\mathrm{V}_{\mathrm{x}}=\mathrm{V}_{\mathrm{y}}=\mathrm{V}_{\mathrm{z}}=0.65 \mu \mathrm{m}$. On the right is the same image with the silicon particles highlighted blue. (B) BSE image obtained from electrode material. The different grey values correspond to the different phases, mainly graphite, pores, binder and Si particles. The ROI is $34 \times 20 \times 20 \mu \mathrm{m}^{3}$ and the voxel sizes are $\mathrm{V}_{\mathrm{x}}=\mathrm{V}_{\mathrm{y}}=8 \mathrm{~nm}, \mathrm{~V}_{\mathrm{z}}=16 \mathrm{~nm}$. (C) XCT image of the pristine electrode. At the bottom is the copper substrate and on top is the anode material. The ROI is about $1.5 \times 1.0 \times 0.04 \mathrm{~mm}^{3}$ and the voxel size is $\mathrm{V}_{\mathrm{x}}=\mathrm{V}_{\mathrm{y}}=\mathrm{V}_{\mathrm{z}}=0.770 \mu \mathrm{m}$. 